\title{
Pre-study plan: Anti-Competitive Entry. Theory and Evidence from a Lab Experiment
}

\author{
Tom-Reiel Heggedal, Leif Helland†, Espen R. Moenªnd Edgar Preugschat ${ }^{\ddagger}$
}

\begin{abstract}
In this paper we study anti-competitive effects of entry within a search framework, and test the implications in an experiment. The starting point for our theoretical analysis is the search model of Varian (1980). In this model, sellers set prices independently and simultaneously. Buyers are either informed about the price quotes or not. All the informed buyers visit the seller with the lowest price quote. The uninformed buyers visit the sellers at random, and buy as long as the price quote is no higher than their reservation value. In equilibrium, sellers randomize over prices, and as the fraction of uninformed buyers goes to zero, the equilibrium expected transaction price converges to zero. Within this model framework, we introduce an entrant. The entrant sets her price after observing the price quotes of the other sellers. In the only symmetric equilibrium of the model, the incumbents set their price equal to the reservation price of the buyers, while the entrant undercuts this price slightly. We test the predictions in the lab. In a pilot study we observe prices that are broadly consistent with our theoretical findings.
\end{abstract}

\section{Introduction}

It is a widely held view that entry of seller will tend to reduce equilibrium prices. In this paper we show, within a model of retail market search, that this may not be the case if the entry of an agent is know ex ante, and the entrant can set its price after observing the price quotes of the incumbent sellers. We then test the model predictions in the lab.

The starting point for our theoretical analysis is the search model of Varian (1980). In this model, sellers set prices independently and simultaneously. Buyers are either informed about the price quotes or not. All the informed buyers visit the seller with the lowest price quote. The uninformed buyers visit the sellers at random, and buy as long as the price quote is no higher than

\footnotetext{
${ }^{*}$ Economics Department \& CESAR, BI Norwegian Business School.

${ }^{\dagger}$ Economics Department \& CESAR, BI Norwegian Business School.

‡Economics Department \& CESAR, BI Norwegian Business School.

$\S$ Department of Economics, Technical University Dortmund \& CESAR, BI Norwegian Business School
} 
their reservation value. In equilibrium, sellers randomize over prices, and as the fraction of uninformed buyers goes to zero, the equilibrium price converges to zero.

Within this model framework, we introduce an entrant. The entrant sets her price after observing the price quotes of the other sellers. In the only symmetric equilibrium of the model, the incumbents set their price equal to the reservation price of the buyers, while the entrant undercuts this price slightly. This holds independently of the number of sellers and the fraction of the buyers that are uninformed (as long as there is at least one uninformed buyer). Hence the introduction of one single entrant may completely change the role of competition, and lead to monopoly prices, even though the equilibrium without entry may be arbitrarily close to the competitive outcome. An asymmetric equilibrium cooexists. In this equilibrium one of the incumbents sets a price that makes undercutting unprofitable while the other incumbent and the entrant set their prices at the reservation value of the buyers.

We test the predictions in a controlled laboratory experiment. In a pilot study we observe prices that are broadly consistent with our theoretical findings. In particular we observe a significant hike in prices when allowing for entry, all else constant.

The pre-study plan is posted at the RCT registry of the AEA.

\section{Model}

There is $U$ uninformed buyers, $N$ informed buyers, and $S$ sellers. Sellers set their price quotes simultaneously and independently. The shadow value of a unit of the good to the seller is zero. The willingness to pay for the good for the buyers is 1 . Sellers are risk neutral.

\subsection{One entrant}

There are a total of $S$ sellers in the economy. One of the $S$ sellers is an entrant. We assume that $S>2$. The sellers that are not entrants are named incumbents. The entrant is different from the incumbents in that it sets its price after it has observed the price quotes of the incumbent. The timing of the game can be summarized as follows:

1. The incumbents set their own price simultaneously and independently.

2. The entrant observes the $S-1$ prices set by the incumbents, and then sets its own price.

3. The informed buyers buy at the firm with the lowest price. If more than one seller sets the lowest price, the buyers visit each of them with the same probability. The uninformed buyers choose a firm to buy from at random. 
If the price of the entrant is the lowest, it gets all the customers. If it is not, it attracts only $U / S$ customers (in expectation).

The game is solved backwards. Let $p^{i}$ denote the lowest price quote set by any of the incumbents at stage 1 . Suppose $p^{i}>p^{0}$. By the definition of $p_{0}$, we know that the entrant is indifferent between setting $p=1$ and sell to uninformed buyers only, and set $p=p_{0}$ and sell to all the informed customers as well. Hence if the entrant can set a higher price than $p_{0}$ and attract all the informed buyers, that must be better than setting $p_{0}$ or 1 . Hence the optimal action for the entrant is to set $p=p^{i}-\varepsilon>p_{0}$ and get all the informed customers.

Suppose then that $p^{i}=p_{0}$. Then the entrant, if it sets $p^{e}=p_{0}$, will receive half of the informed customers in expectation. If the entrant obtains all the informed customers, it is indifferent by setting $p_{0}$ and 1 . It follows that the best response of the entrant then is to set $p=1$. The same is true for $p^{i} \leq p_{0}$. Hence the optimal response of the entrant is as follows:

- If $p^{i} \in\left(p_{0}, 1\right)$, set $p^{e}=p^{i}-\varepsilon$

- If $p^{i} \leq p_{0}$, set $p^{e}=1$.

Then consider the incumbent. We want to show that given the entrant's optimal strategy, it is an optimal strategy for all the incumbents to set $p=1$. Suppose an incumbent deviates and set $p \in\left(p_{0}, 1\right)$. The incumbent will be underbid by the entrant, and not get any informed customers. Hence it is strictly better to set the price equal to 1 . Suppose an incumbent deviates and set $p=1$. Then the entrant sets $p^{e}=1$, and the incumbent is indifferent between setting $p_{0}$ and $p_{1}$.

Proposition 1 The game has a unique symmetric equilibrium, in which the equilibrium outcome is that the incumbents set $p=1$ and the entrant sets $p=$ $1-\varepsilon$.

We have already shown that the outcome is an equilibrium outcome. We only have to show that the equilibrium is the only symmetric equilibrium. Suppose that there exists another symmetric equilibrium in which the incumbents' strategy is given by the distribution function $F^{i}$. Suppose $p^{\prime} \in\left(p_{0}, 1\right)$ is in the support of $F^{i}$. If a seller sets $p^{\prime}$, it will either be outbidden by one of the other incumbents or by the entrant, and it will only sell to uninformed buyers. Hence $p^{\prime}$ is strictly dominated by $p=1$. It follows that $F^{i}$ cannot have any mass on the interval $\left(p_{0}, 1\right)$. Then consider $p_{0}$. Suppose the incumbents set $p_{0}$ with probability $\alpha>0$. When they play $p_{0}$, there is a probability $1-(1-\alpha)^{S-1}>0$ that another incumbent also sets $p_{0}$, in which case the pay-off obtained is strictly lower than the pay-off obtained by setting $p=1$ for any $\alpha>0$.. Hence $\alpha=0$. By construction of $p_{0}$, it cannot be optimal for the incumbent to set $p<p_{0}$. This completes the proof.

However, there exist other asymmetric equilibria. Suppose one of the incumbents sets $p=p_{0}$ and all the other incumbents set $p=1$. Then the entrant's 
best response prescribes to set $p=1$. The incumbent that sets $p=p_{0}$ is indifferent between setting $p=1$ and $p=p_{0}$. Hence this is also an equilibrium outcome.

\section{$2.2 \quad E$ entrants}

Suppose now that there are $E$ entrants among the $S$ sellers. We first focus on symmetric equilibria.

We first derive the optimal response of the entrants. Suppose $p^{i}=1$. The entrants know that they can outbid all the incumbents by setting $p^{e}<1$. However, there is also competition between the entrants, who can undercut each-other. By using a standard argument, it follows that the entrants play with mixed strategies. Let $F^{e}(p ; 1)$ denote the mixed strategy used by all entrants (denoted $F^{e}(p)$ from now on). It is straight-forward to show that $F^{e}$ has support $\left(p_{0}, 1\right)$ and is without mass points. The seller at the top of the support only sells to uninformed agents, and thus gets a sale of $U / S$. Hence the expected profit at the top of the distribution, and hence for all the sellers, is equal to the profit if setting $p=1$, analogous to what we found above. Hence for all $p$ in the support of $F^{e}$, we have that

$$
\frac{U}{S} p+\left(1-F^{e}(p)\right)^{E-1} p N=\frac{U}{S}
$$

Or that

$$
F^{e}(p)=1-\left(\frac{U}{S N} \frac{1-p}{p}\right)^{\frac{1}{E-1}}
$$

Suppose then that $p^{i} \in\left(p_{0}, 1\right)$. Again, undercutting implies that there must exist a continuous distribution $F^{e}\left(p ; p^{i}\right)$ below $p^{i}$. Suppose all the entrants were randomizing prices below $p^{i}$. The seller at the top of the support would only sell to the uninformed buyers at the price of $p^{i}$. Hence the seller would be better off setting $p=1$. It follows that this cannot be a best response, and that $F^{e}$ has a mass point at 1 . Let $F_{1}$ denote the probability that an entrant sets $p=1$. It follows that the probability that an entrant is the only entrant who does not set $p=1$ is $F_{1}^{E-1}$. It follows that $F_{1}$ is given by $F_{1}^{E-1} p^{i}\left(N+\frac{U}{S}\right)=\frac{U}{S}$, or that

$$
F_{1}=\left(\frac{U}{N S} \frac{1-p^{i}}{p^{i}}\right)^{\frac{1}{E-1}}
$$

Below $p^{i}, F^{e}$ is given by (1).

Suppose then that $p^{i}=p_{0}$. By the same argument as above, it follows that all entrants set $p^{e}=1$.

Consider then the incumbents. Let $F^{i}(p)$ denote the equilibrium distribution of prices chosen by the incumbents. We want to show that the equilibrium distribution $F^{i}(p)$ is degenerate, with all its mass at $p=1$. First, suppose all entrants set $p=1$. If an entrant deviates and sets $p^{\prime} \in\left[p^{0}, 1\right)$, the probability of 
a sale is $\left(1-F^{e}\left(p^{\prime}\right)\right)^{E} \leq\left(1-F^{e}\left(p^{\prime}\right)\right)^{E-1}$. That is, the pay-off to the incumbent is lower than that of the incumbent, with strict inequality except at $p^{\prime}=p_{0}$. Hence the degenerate incumbent distribution is an equilibrium distribution.

The proof of uniqueness is identical to the proof of uniqueness with one entrant. Suppose $p^{\prime} \in\left(p_{0}, 1\right)$ is in the support of $F^{i}$. If an incumbent seller sets $p^{\prime}$, it will either be outbidden by one of the other incumbents or by the entrant, and it will only sell to uninformed buyers. Hence $p^{\prime}$ is strictly dominated by $p=1$. It follows that $F^{i}$ cannot have any mass on the interval $\left(p_{0}, 1\right)$. Then consider $p_{0}$. Suppose the incumbents set $p_{0}$ with probability $\alpha>0$. When they play $p_{0}$, there is a probability $1-(1-\alpha)^{S-1}>0$ that another incumbent also sets $p_{0}$, in which case the pay-off obtained is strictly lower than the pay-off obtained by setting $p=1$ for any $\alpha>0$.. Hence $\alpha=0$. By construction of $p_{0}$, it cannot be optimal for the incumbent to set $p<p_{0}$. This completes the proof.

Proposition 2 Suppose there are $E>1$ entrants and that $S>2$. Then the game has a unique symmetric equilibrium. In this equilibrium, all the incumbents set $p=1$. All the entrants play mixed strategies on the interval $\left(p_{0}, 1\right]$. The price distribution of each seller is given by (1)

In addition, there exist asymmetric equilibria. In particular, there exists an equilibrium in which one incumbent plays $p=p_{0}$, while all the other incumbents and all the entrants play $p=1$.

\subsection{No entrant}

This section sets out the model of Varian to facilitate comparisons with the entry case.

We first look at the case where there are at least some uninformed buyers, $U \geq 1$. The number of sales to uninformed customers is binomially distributed and thus equal to $U / S$ in expectation. The expected sales to informed agents only depend on whether or not the seller's price is lower than the other firms' prices. Thus $\mu^{n, N}\left(p_{s}, p_{-s}\right)=N+U / S$ if $p_{s}$ is the lowest price and $\mu^{n, N}\left(p_{s}, p_{-s}\right)=U / S$ otherwise. ${ }^{1}$ One can show that the symmetric equilibrium entails a mixed strategy given by the c.d.f. $F(p)$ with support $p \in\left[p_{0}, 1\right] .^{2}$ It is convenient to determine the equilibrium strategy by looking at the indifference between the "rip-off" price of 1 and any other price in the support of $F(p)$ : $\int \pi_{s}^{n, N}\left(p_{s}, p_{-s}\right) d F\left(p_{-s}\right)=\int \pi_{s}^{n, N}\left(1, p_{-s}\right) d F\left(p_{-s}\right)$. This can be written as:

$$
\left(U / S+N\left(1-F\left(p_{s}\right)\right)^{S-1}\right) p_{s}=U / S \cdot 1
$$

The left-hand side shows the pay-off when setting a price $p_{s}$. Independent of the price, the seller will sell in expectation to $U / S$ uninformed sellers. If it sets

\footnotetext{
${ }^{1}$ It can be shown that $F(p)$ has no mass points so that ties are a measure zero event. The intuition is that if $F$ had a mass point at $p^{\prime}$, the expected number of sales would increase discretely by lowering the price slightly below $p^{\prime}$, hence advertising $p^{\prime}$ cannot be optimal.

${ }^{2}$ The supremum of the support has to be 1: if a firm knows with certainty that it will only attract uninformed buyers, the optimal price is the reservation value of uninformed buyers.
} 
the lowest price, it will in addition sell to $N$ informed sellers, and this happens with probability $(1-F(p))^{S-1}$. The right hand side shows the expected pay-off when setting $p_{s}=1$. Solving for $F(p)$ gives:

$$
F(p)=1-\left(\frac{1-p}{p} \frac{U}{S N}\right)^{1 /(S-1)} \quad \text { with } p \in\left[p_{0}, 1\right] .
$$

It is straightforward to verify that the lower bound of the support is given by $p_{0}=\frac{U}{U+S N}$. Using the tail formula for the expected value, the expected price can be expressed as:

$$
\begin{aligned}
E[p] & =p_{0}+\int_{p_{0}}^{1}(1-F(p)) d p \\
& =p_{0}+\int_{p_{0}}^{1}\left(\frac{1-p}{p} \frac{U}{S N}\right)^{1 /(S-1)} d p .
\end{aligned}
$$

Notice that this expected price is strictly decreasing in the number of informed buyers as a percentage of uninformed buyers, i.e. $N / U$. The cumulative distribution function of the lowest price in the market is given by $1-(1-F(p))^{S}$. By using the tail formula again it follows that the expected minimum price at which the informed buyers purchase the good is given by:

$$
E\left[p_{\min }\right]=p_{0}+\int_{p_{0}}^{1}\left(\frac{1-p}{p} \frac{U}{S N}\right)^{S /(S-1)} d p
$$

\section{Experimental design}

Our core experiment is built on a $2 \times 3$ factorial design. The first dimension is whether there is entry or not. The second dimension is the share of uninformed buyers: $10 \%, 30 \%$ and $60 \%$. The number of sellers and buyers is kept constant. In all treatments there are 3 sellers and 100 buyers. In the entry-treatments, two of the sellers are incumbents while the last seller is the entrant. While all sellers are humans all buyers are robots. Unique subjects are used in all sessions. Sellers are randomly re-matched in each period from matching groups of 9 subjects. Prices are constrained to the interval 0-100, and can be set with up to three decimals.

As an independent observation we regard the average price in a matching block over the 60 games played. The table below show the expected prices in our six treatments when the symmetric equilibrium is played (rounded to the nearest integer).

\begin{tabular}{llll}
\cline { 2 - 4 } & \multicolumn{3}{c}{ Pct uninformed } \\
Entry & $15 \%$ & $30 \%$ & $60 \%$ \\
\cline { 2 - 4 } No-entry & 100 & 100 & 100 \\
\cline { 2 - 4 } & 32 & 46 & 68 \\
\cline { 2 - 3 }
\end{tabular}

Table 1: Expected prices in the symmetric equilibrium 


\section{Pilot experiment and power tests}

Based on the observed treatment effect and the variation in the matching blocks of the pilot we calculate the number of independent observations needed to reach a power of $90 \%$ or better using a non-parametric (Wilcoxon rank) test.

The pilot was carried out at the BI research lab, using subjects recruited from the general student populations of BI and the University of Oslo. For the pilot we used two matching groups in the entry treatment and two matching groups in the no-entry treatment. The number of uninformed buyers were set at 30 in both treatments.

\subsection{Pilot results}

The average price observed in the entry treatment of the pilot was 82.2 , undershooting the expected price in the symmetric equilibrium with almost 20 percentage points. Of the 120 markets in this treatment we observe price configurations close to or at the symmetric equilibrium in $46 \%$ of the markets. In $13 \%$ of the markets we observe price configurations close to or at the asymmetric equilibrium. In $2 \%$ of the markets both incumbents are at or close to $p_{0}=12.5$ while the entrant sets her price at the reservation value of 100 , indicating a failure to coordinate on the asymmetric equilibrium. Thus, $61 \%$ of markets in the entry treatment are rationalizable within the model. Entrant behavior is almost always a best-reply to the observed incumbent prices.

The average price in the no-entry treatment was 57.0, overshooting the expected price in the symmetric equilibrium with a full 11 percentage points. However, less than $\frac{1}{4} \%$ of observations are below $p_{o}$ in this treatment, demonstrating that subjects are setting prices that are not inconsistent with mixing on the appropriate support.

\subsection{Power tests}

Based on the observed treatment effect and the variation in the matching blocks of the pilot we calculate the number of independent observations needed to reach a power of $90 \%$ or better using a non-parametric (Wilcoxon rank) test and setting the significance level at $5 \%$.

The average price per block and treatment, and the variance of price per treatment are displayed in the table:

\begin{tabular}{lcccc}
\hline & \multicolumn{2}{c}{ Entry } & \multicolumn{2}{c}{ No-Entry } \\
Block & Mean & Variance & Mean & Variance \\
\hline 1 & 84.4 & - & 54.7 & - \\
2 & 77.9 & - & 56.3 & - \\
\hline 3 & 91.1 & - & 61.9 & - \\
\hline 4 & 75.6 & - & 55.1 & - \\
\hline Total & 82.3 & 48.7 & 57.0 & 11.1 \\
\hline
\end{tabular}


Based on these numbers and using the simulation software of Bellmare et al. (2014) we obtain a power of $100 \%$ with 4 blocks per treatment. Expecting more moderate treatment effects for $60 \%$ uninformed robot buyers, and wanting to cut down on the risk of false positives, we double the number of matching blocks from four to eight for all six treatments of the experiment.

\section{Conclusion}

Overall, the pilot supports the main message of the model: That entry in the face of uninformed buyers drive prices towards monopoly levels relative to the absence of entry. The treatment effect in the pilot is a full 23 percentage points. Going forward with our design, will clarify whether this treatment difference is shrinking in the number of uninformed buyers, and whether prices in the entry treatment stay consistently high as the number of uninformed buyers is decreased.

The full experiment should reveal whether there are systematic and substantial deviations from the equilibria of the model. Such deviations may suggest modifications of the model that can explain the data better. This will be a central part of the project. A full set of data on the core experiment will be collected in April 2019.

\section{References}

Varian, Hal R. "A model of sales." The American Economic Review 70.4 (1980): 651-659. 